\title{
Transgenic Mosquitoes to Control Vector Borne Diseases
}

Sampath LDS ${ }^{1}$, Gunawardene YINS $^{2}$ and Dassanayake RS ${ }^{1^{*}}$

${ }^{1}$ Department of Chemistry, University of Colombo, Colombo 03, Sri Lanka

${ }^{2}$ Molecular Medicine Unit, Faculty of Medicine, University of Kelaniya, Ragama, Sri Lanka

*Corresponding author: Dassanayake RS, Department of Chemistry, University of Colombo, Colombo 03, Sri Lanka, Tel: + 94-11-2503 367; E-mail: rsdassanayake@chem.cmb.ac.lk

Rec date: May 12, 2015; Acc Date: May 15, 2015; Pub date: May 19, 2015

Copyright: (c) 2015 Sampath, et al. This is an open-access article distributed under the terms of the Creative Commons Attribution License, which permits unrestricted use, distribution, and reproduction in any medium, provided the original author and source are credited.

\section{Mini-Review}

The mosquito borne diseases have achieved the world wide attention due to the high number of cases and deaths reported to WHO annually. Such diseases include malaria and dengue fever. Control of these diseases has been difficult due to drug resistance in parasites (e.g. malaria), insecticide resistance in mosquitoes, lack of effective vaccines and failure of the vector control strategies to minimize human-mosquito contact. As a novel approach for the rising situation, mosquito population replacement strategies and, genetic based mosquito population suppression and elimination strategies such as Release of Insects carrying a Dominant Lethal (RIDL) have been introduced. Both strategies used for the development of transgenic mosquitoes (TMs) to minimize/eliminate the mosquito borne diseases. In this review the development of TMs and their role in controlling diseases is described.

For the development of transgenic mosquitoes suitable vector transformation systems, a DNA construct having a reporter gene and pathogen effector molecule which is driven by a strong promoter are required. The vector transformation systems used to produce transgenic mosquitoes include virus transducing systems, transposable elements, and endosymbionts. The virus transducing systems include double sub genomic Sindibs virus (SIN) expression vector TE/3'J and SIN replicon. With the help of these viruses transducing systems such as Aedes, Anopheles as and Culex mosquitoes could be transformed. For an example, transgenic Ae. aegypti mosquitoes have been developed to demonstrate the efficiency of antisense RNA and their ability to block dengue, yellow fever and LaCross virus transmission with the help of SIN virus transducing systems. Even though virus transducing systems were capable of transforming and integrating transgenes into mosquito genome, they were not capable of spreading the transgene efficiently to mosquitoes because the transgene was inherited to the next generation via Mendelian inheritance only. For the efficient transgene spreading in the natural population, an enormous numbers of mosquitoes were required. In order to produce such numbers of TMs, transposable elements (TE) and endosymbionts that were capable of spreading the transgene in a non-Mendelian manner was begun to use. The transposable elements used to transform Ae. aegypti were Hermes [1] from housefly Musca domestica, mariner element Mos1 [2] from D. mauritiana, piggyback (pBac) [3] from Trichoplusiani, and minos from D. hydei. The vector transformation could also be done with the help of endosymbionts such as intracellular symbiotic bacterium Wolbachia [4]. Genetically modified Wolbachia could be used to transduce mosquitoes with parasite transmission blocking genes. For an example, Ae. aegypti infected with wMelPop-CLA Wolbachia strain has shown to reduce the lifespan of adult mosquitoes resulting in the reduced potential of dengue virus type2 (DENV-2) transmission. Another strain named wMel Wolbachia strain also was used to block the transmission of
DENV-2 in Ae. Aegypti [5]. In the past, insecticide resistance genes and antibiotic resistance genes were used as reporter genes when TMs were developed but currently, fluorescent markers such as enhanced green fluorescent protein (EGFP) [2,6] and Discosoma sp. Red fluorescent protein (dsRED) $[7,8]$ are used to select transgenic mosquitoes from non-transgenic mosquitoes. Using fluorescent markers as reporter genes saved the time and effort of rearing nontransgenic mosquitoes to adults because the fluorescent protein expressing mosquitoes could be observed and separated even in early larval stages, however, if insecticide resistance genes were used as reporter genes both transgenic and non-transgenic larvae had to be reared until adult stage. 4 Some of the pathogen effector molecules used when producing transgenic mosquitoes were antiparasitic peptides3, antimicrobial peptides [9], antisense RNA, and shRNA [2]. As promoters for the DNA constructs, salivary gland specific promoters from Maltase-like I gene (Mall) and Apyrase gene (Apy) of $A$ e. aegypti had been used to drive the transgene expression in transgenic Ae. aegypti in the past. Since the transgene expressions driven by these promoters were very weak, stronger promoters were subsequently used in developing TMs [4]. The strong promoters such as Ae. aegypti carboxypeptidase A $(A e C P A)$ [2] and Vitellogenin ( $V g)$ [9] genes promoters that drives expression in stage, tissue and sex specific manner, Anopheles gambiae carboxy peptidase $(A g C P)$ [3] and $\beta 2$ [10] genes promoters, Ae. aegypti Act5C [7] and Actin-4 (AeAct-4)8 genes promoters have been used to drive the transgene expression in strong manner in TMs. In addition, the $h s p 70$ promoter as one of the minimal promoters has also been used [7]. The transgenic mosquitoes produced using above mentioned genetic elements to combat against major mosquito borne diseases such as malaria and dengue are described below.

The World Health Organization (WHO) reports 135-287 millions of malaria cases annually [11] indicating urgent need for new control strategies. TMs as one of the promising control strategies, the mosquito having a foreign DNA that codes for a synthetic gene, $\mathrm{AgCP}[\mathrm{SM} 1]$ [4] containing CP signal sequence, and coding sequences of four subunits of SM1 under the regulation of $\mathrm{AgCP}$ promoter was constructed and inserted into pBac transposable element (TE) containing vector with enhanced green fluorescent protein (EGFP)gene under the regulation of mosquito eye specific $3 \mathrm{xP} 3$ promoter. The final DNA construct was then transformed along with transposase enzyme coding helper plasmid into embryos of $A n$. stephensi and EGFP expression in the mosquito eyes was then used for the identification of TMs. The induction of $A g C P$ promoter of the construct to express SM1 tetramer protein is generally taking place for approximately 24 hours following ingestion of blood meals by the mosquitoes, An. stephensi and CP signal peptide of this protein direct SM1 to be secreted into the midgut to cover its luminal surface to inhibit the invasion of parasite Plasmodium to the epithelium of 
midgut, which happen at the same time period of SM1 protein expression resulting in fewer parasite invasions to salivary glands making less parasites in the duct lumen for transmission. The TMs developed in this manner will pass on the construct to future generations replacing the wild type mosquitoes. Therefore, the transmission of the malaria parasite can be curtailed significantly. However, the emergence of resistant parasite through natural selection due to genetic diversity and mutability of Plasmodium has been the issue of concern. As a solution for this, the modification of mosquito genomes with multiple genes that could inhibit the parasite development by different mechanisms were carried out resulting mosquitoes called transgenic hybrid mosquitoes that over expresses antimicrobial peptides (AMP) such as Cecropin A and Defensin A under Vg promoters, after a blood meal in the haemolymph of female mosquitoes. The coordinated and cooperative action of these AMP has been shown to completely block the transmission of Plasmodium gallinaceum by Ae. aegypti. However, this strategy has not worked for malaria vector, An. gambiae having infection of either $P$. falciparum or $P$. berghei as a result of non activation of defensins or cecropins in the latter mosquitoes. However, based on this result it was concluded that combinatorial over expression of several antiparasitic effector molecules in TMs would completely block the transmission of Plasmodium in Anopheles genus mosquitoes to prevent malaria [9].

Genetics-based population suppression and elimination strategies that distort sex ratio of the natural population of An.gambiae using TMs were applied to control malaria disease. In this strategy, a DNA construct designed as $\mathrm{pBac}\{3 \times \mathrm{xP} 3-\mathrm{DsRed}\} \mathrm{b} 2-\mathrm{eGFP}: \mathrm{I}-\mathrm{PpoI}$ was transformed into the embryos of Ae. aegypti and the resultant TM was then identified using red colour fluorescence in the eyes as a result of expression of DsRed gene. The promoter $\beta 2$ of this construct that drives the expression of the fusion protein, eGFP::I-PpoI homing endonuclease is activated only in male anopheline TMs' gonads, before the first meiotic division and then throughout subsequent division until fully differentiation of spermatozoa. This I-PpoI homing endonuclease is capable of selectively cleaving the $28 \mathrm{~S}$ rDNA on chromosome $\mathrm{X}$ of An. gambiae mosquitoes resulting in nuclear fragmentation and cell death and thus increasing male biased sex ratio and decreasing female mosquitoes in the population leading gradual extinction of mosquito population. Spermatozoa produced by a mosquito even with partial inheritance (i.e. mating TM and a wild type mosquito) of transgene capable of destroying the $\mathrm{X}$ chromosomes of spermatozoa as all the spermatozoa derived from a single spermatogonial cell are connected through cytoplasmic bridges allowing the sharing of cytoplasmic constituents including I-PpoI homing endonuclease enzyme between spermatozoa. pBac, 3xP3 promoter and DsRed sequences in this construct have the same functions as described elsewhere and the terminator sequence used in this construct was derived from the $\beta 2$ gene of anopheline mosquitoes [10]. It has been shown the large scale laboratory experiments conducted using these TMs have produced successful results in controlling mosquito populations [12,13].

Dengue disease is another major global problem and the WHO reports 50-100 million dengue infections occur annually [14,15] yet indicating the importance of effective control strategies to contain this disease. As TMs is one of the promising strategies to control the dengue virus transmission, mosquito genome carrying a foreign DNA sequence named, pMos-carb/Mnp+/i/Mnp-/svA was developed to provide the resistant to dengue virus type 2 (DENV-2) replication in Ae. aegypti. This mosquito has been genetically modified in such way that it to express $\mathrm{Mnp}+/ \mathrm{i} / \mathrm{Mnp}$ - region following ingestion of blood meals through the induction of $A e C P A$ promoter. As Mnp+ and Mnpare in sense and antisense orientations, the transcribed RNA molecule essentially forms a double stranded RNA (dsRNA) in the mosquito midgut [2] and this dsRNA then activates a RNA interference pathway [16] resulting in the degradation of Mnp region of the DENV-2 genome making pathogen incompetent $A$. aegypti vector [2]. The role of svA sequence in the construct is to functions as the polyadenylation signal as in the Simian virus 40 VP1 gene. TMs developed in this manner will passed on the construct to future generations replacing the wild type mosquitoes with TMs. These TMs were resistant to DENV-2 but not to DENV-4 suggesting that the refractoriness obtained here is specific to DENV-2. However, DENV transmission by Ae. aegyptican be reduced using this approach and the TE used in this construct was mariner MosI. The $3 \mathrm{xP} 3$ promoter and EGFP gene have the same function as described above.

A genetics-based population suppression and elimination approach such as RIDL is yet another strategy and provides a better control to vector borne diseases including dengue disease. This was achieved by transforming the embryos of Ae. aegypti with the plasmid vector designated pLA513 containing pBacTE, tTAV gene (tetracyclinerepressible transcriptional activator) under the tetO7 tTAV binding site, $h s p 70$ minimal promoter and DsRed2 gene under thorax specific Act5C promoter. In the absence of tetracycline medium, tTAV protein binds to tetO sequence and express more tTAV protein which is toxic only in high level to insects due to interaction of its VP16 domain with key transcription factors in mosquitoes [7]. Further, high levels of tTAV results in the disruption of ubiquitin dependent protein degradation system [8]. However, the larve or adult mosquitoes in a medium containing tetracycline, this toxicity and degradation system was completely suppressed due to binding tetracycline with tTAV protein and thus tTAV protein is unable to bind the tetO sequence affecting high level expression of tTAV protein that required for the toxicity. Therefore, when homozygous adult male mosquitoes (LA513A) reared in a medium containing tetracycline are released into the environment, they would mate with wild type female mosquitoes and in the absence of tetracycline medium in the environment these transgenic adult mosquito progeny will die due to accumulated tTAV toxicity and disruption of ubiquitin dependent protein degradation system [7]. The release of TMs in this manner to a jungle area of Malaysia proved that the life expectancy of TM was as short as 1.8-2.2 days [17]. The larvae produced, as a result of the mating between TMs and wild type mosquitoes, compete with wild type larvae for the food in the medium and finally wild type larvae will die due to competition for the food while transgenic larvae will die due to accumulated tTAV toxicity. As a result Ae aegypti mosquito population can be suppressed or eliminated and hence this strategy provides a way to control not only the transmission of dengue virus but also the virus that caused yellow fever. The open field release of these type of TMs in Grand Cayman Island as well as in Brazil have shown successful results [12].

Using a similar strategy to that of to RIDL technique, a flightless phenotype of $A$ e. aegypti was developed. In this approach too, a new DNA construct designated, AeAct4-tTA was engineered and transformed into the genome of the preblastoderm embryos of $A e$. aegypti and the TMs thus produced in this manner displayed red fluorescence in the indirect flight muscles (IFMs) of female larvae, pupae and adults when reared in non tetracycline medium. The promoter AeAct-4 in this construct is active in female TMs and drives the expression of tTAV mainly in IFM and the binding of tTAV to the tetO sequence in the responsive element, tRE of the DNA construct 
drives the expression of reporter gene DsRed resulting red fluorescence in the IFMs of female larvae, pupae and adults when they reared in nontetracycline medium. The concurrent expression of tTAV causes the toxicity to these mosquitoes as described elsewhere resulting in flightlessness which is similar to lethality achieved in RIDL approach. The flightless adult mosquitoes will have a reduced ability to escape water after emerging, increased vulnerability to predators around breeding sites as well as the inability to fly to locate a blood meal and mate [8]. As a result, Ae. aegypti mosquito population can be suppressed or eliminated resulting the control of Dengue and yellow fever transmission by Ae. aegypti mosquitoes as in previous case.

Finally, vector borne diseases caused by mosquitoes has been a threat to billions of human life around the globe. In such setting, the development of TMs that have impaired competency to transmit pathogens or population suppression and elimination ability as result of alteration of genetics of mosquitoes is a promising approach that could potentially reduce threats exerted by mosquitoes to humans. The work carried out so far on TMs has shown successful results in the laboratory and open field release experiments indicating TMs to be the leading strategy in the future to control mosquito borne diseases.

\section{References}

1. Jasinskiene N, Coates CJ, Benedict MQ, Cornel AJ, Rafferty CS, et al. (1998) Stable transformation of the yellow fever mosquito, Aedes aegypti, with the Hermes element from the housefly. Proc Natl Acad Sci U S A 95: 3743-3747.

2. Franz AW, Sanchez-Vargas I, Adelman ZN, Blair CD, Beaty BJ, et al. (2006) Engineering RNA interference-based resistance to dengue virus type 2 in genetically modified Aedes aegypti. Proc Natl Acad Sci U S A 103: 4198-4203.

3. Ito J, Ghosh A, Moreira LA, Wimmer EA (2002) Transgenic Anopheline Mosquitoes Impaired in Transmission of a Malaria Parasite. Nature, 417: 452-455.

4. Sperança MA, Capurro ML (2007) Perspectives in the control of infectious diseases by transgenic mosquitoes in the post-genomic era--a review. Mem Inst Oswaldo Cruz 102: 425-433.
5. Walker T, Johnson PH, Moreira LA, Iturbe-Ormaetxe I, Frentiu FD, et al. (2011) The wMel Wolbachia strain blocks dengue and invades caged Aedes aegypti populations. Nature 476: 450-453.

6. Kokoza V, Ahmed A, Wimmer EA, Raikhel AS (2001) Efficient Transformation of the Yellow Fever Mosquito Aedes Aegypti Using the piggyBac Transposable Element Vector pBac[3xP3-EGFP Afm]. Insect Biochem Mol Biol 3: 1137-1143.

7. Phuc HK, Andreasen MH, Burton RS, Vass C, Epton MJ, et al. (2007) Late-acting dominant lethal genetic systems and mosquito control. BMC Biol 5: 11.

8. Fu G, Lees RS, Nimmo D, Aw D, Jin L, et al. (2010) Female-specific flightless phenotype for mosquito control. Proc Natl Acad Sci U S A 107: 4550-4554.

9. Kokoza V, Ahmed A, Woon Shin S, Okafor N, Zou Z, et al. (2010) Blocking of Plasmodium transmission by cooperative action of Cecropin $A$ and Defensin A in transgenic Aedes aegypti mosquitoes. Proc Natl Acad Sci U S A 107: 8111-8116.

10. Windbichler N, Papathanos PA, Crisanti A (2008) Targeting the X Chromosome during Spermatogenesis Induces $\mathrm{Y}$ Chromosome Transmission Ratio Distortion and Early Dominant Embryo Lethality in Anopheles Gambiae. PLoS Genet 4: e1000291

11. World Health Organization. World Malaria Report 2013, 2013

12. Gabrieli P, Smidler A, Catteruccia F (2014) Engineering the control of mosquito-borne infectious diseases. Genome Biol 15: 535

13. Klein TA, Windbichler N, Deredec A, Burt A, Benedict MQ (2012) Infertility resulting from transgenic I-PpoI male Anopheles gambiae in large cage trials. Pathog Glob Health 106: 20-31.

14. http://www.who.int/csr/disease/dengue/impact/en/

15. World Health Organization (2012) Treatment, Prevention and Control Global Strategy for Dengue Prevention and Control 2: 1-5.

16. Sánchez-Vargas I, Scott JC, Poole-Smith BK, Franz AW, BarbosaSolomieu V, et al. (2009) Dengue virus type 2 infections of Aedes aegypti are modulated by the mosquito's RNA interference pathway. PLoS Pathog 5: e1000299.

17. Lacroix R, McKemey AR, Raduan N, Kwee Wee L, Hong Ming W, et al. (2012) Open field release of genetically engineered sterile male Aedes aegypti in Malaysia. PLoS One 7: e42771. 